\title{
Improving Safety Structures on Sprint and Midget Race Cars
}

\author{
Grant Hankins, Kenny Krajnik, Brad Galedrige, Shahab Sakha \\ Motorsports Engineering Students
}

\author{
Pete Hylton, Wendy Otoupal \\ Motorsports Engineering Faculty
}

Indiana University-Purdue University Indianapolis

A number of performance and safety related aspects of motorsports have begun to receive increased attention in recent years, using the types of engineering analysis common to other industries such as aerospace engineering. As these new engineering approaches have begun to play a larger role in the motorsports industry, there has been an increase in the use of engineering tools in motorsports design and an increase in the inclusion of motorsports in the engineering education process. The design, modeling, and analysis aspects of roll cages for American short-track open-wheel racing cars is the objective of this project. Roll cage structures were initially integrated into cars of this type in the 1960s. Countless lives have been saved and serious injuries prevented since the introduction of cages into these types of cars. However, the general configuration of these cages has not seen significant change or improvement in the four decades since their introduction. Crash tests conducted in the past two years have demonstrated the potential for the driver's helmet to impact an external barrier and also indicated that some tubes and joints in the cage structure may be excessively loaded in certain crash scenarios. Recent innovations in engineering design techniques involving the utilization of Three Dimensional (3D) Finite Element Modeling (FEM) and Finite Element Analysis (FEA) have opened opportunities for engineering analysis which could improve the design of these roll cages. This project utilizes research, modeling, and analysis approaches from academia to assist in the design of new aspects of competition vehicles aimed at increasing safety. 